\title{
An Application of Quantum Annealing Computing to Seismic Inversion
}

\author{
Alexandre M. Souza ${ }^{1 *}$, Eldues O. Martins ${ }^{2}$, Itzhak Roditi ${ }^{1,3}$, Nahum Sá $^{1}$, Roberto S. Sarthour $^{1}$ \\ and Ivan S. Oliveira ${ }^{1}$ \\ ${ }^{1}$ Centro Brasileiro de Pesquisas Físicas, Rio de Janeiro, Brazil, ${ }^{2}$ Petróleo Brasileiro S.A., Centro de Pesquisas Leopoldo Miguez \\ de Mello, Rio de Janeiro, Brazil, ${ }^{3}$ Institute for Theoretical Physics, Zurich, Switzerland
}

Quantum computing, along with quantum metrology and quantum communication, are disruptive technologies that promise, in the near future, to impact different sectors of academic research and industry. Among the computational challenges with great interest in science and industry are the inversion problems. These kinds of numerical procedures can be described as the process of determining the cause of an event from measurements of its effects. In this paper, we apply a recursive quantum algorithm to a D-Wave quantum annealer to solve a small scale seismic inversions problem. We compare the obtained results from the quantum computer to those derived from a classical algorithm. The

OPEN ACCESS

Edited by:

Adam Miranowicz,

Adam Mickiewicz University, Poland

Reviewed by:

Natalia Korolkova,

University of St Andrews,

United Kingdom

Che-Ming Li,

National Cheng Kung University,

Taiwan

*Correspondence:

Alexandre M. Souza amsouza@cbpf.br

Specialty section:

This article was submitted to

Quantum Engineering and

Technology,

a section of the journal

Frontiers in Physics

Received: 27 July 2021

Accepted: 06 December 2021

Published: 18 January 2022

Citation:

Souza AM, Martins EO, Roditi I, Sá N, Sarthour RS and Oliveira IS (2022) An Application of Quantum Annealing

Computing to Seismic Inversion.

Front. Phys. 9:748285.

doi: 10.3389/fphy.2021.748285 accuracy achieved by the quantum computer is at least as good as that of the classical computer.

Keywords: quantum computing, quantum annealing, seismic inversion, linear equations, binary optimization

\section{INTRODUCTION}

Seismic geophysics relies heavily on subsurface modeling based on the numerical analysis of data collected in the field. The computational processing of a large amount of data generated in a typical seismic experiment can take an equally large amount of time before a consistent subsurface model is produced. Electromagnetic reservoir data, like CSEM (Controlled Source Electromagnetic), petrophysical techniques, such as electrical resistivity and magnetic resonance on multi-wells, and engineering optimization problems like reservoir flux simulators, well field design and oil production maximization also need a strong computational apparatus for analysis.

On the other hand, in the past decade, there has been much progress in the development of quantum computers: Machines exploiting the laws of quantum mechanics to solve hard computational problems faster than conventional computers. A concrete example of such progress is the so-called quantum supremacy, that has been recently demonstrated using specific purpose quantum computers [1-3]. The Geoscience field and related industries, such as the hydrocarbon industry, are strong candidates to benefit from those advances brought by quantum computing.

Currently, different quantum technologies and computational models are being advanced. Giant companies like IBM, Google, and Intel are developing quantum computers based on superconducting technologies [4]. Other companies are also putting considerable effort into building a fully functional quantum computer based on Josephson junctions, such as the North American Rigetti, whereas, the also American IonQ and the Austrian AQT are working on computers based on trapped ions [5]. The Canadian company D-Wave, leader in the computational model known as quantum annealing [6], is already trading quantum machines, and the also Canadian Xanadu is providing cloud access to their photonic quantum computer $[7,8]$. 
Recent reviews comparing superconducting and trapped-ion technologies and different cloud-based platforms can be found in Refs. [9, 10], respectively.

In the field of Geoscience, recent works have used quantum annealers to hydrology inversion problems [11, 12]. In those works, it was shown that, although the size of the problem that can be solved on a third-generation D-Wave quantum annealer is considered small for modern computers, they are larger than the problems solved with similar methodology with Intel's third and fourth generation chips. It is also important to mention that optimization techniques are widely used in seismic inversions, but usually classical algorithms get stuck in local minima. Previous works [13-15] have indicated that quantum annealing can be advantageous to solve seismic problems. However, the potential applications of quantum computing in Geoscience has so far been largely unexploited in the specialized literature.

In this work, we present a formulation of a seismic problem as a binary optimization, and one small scale subsurface seismic problem is solved using the D-Wave quantum annealer available in the Amazon Braket service. We have evaluated the performance of the quantum computer comparing the results obtained to those derived from a classical computer. Our analysis was focused on the accuracy. It was found that the accuracy achieved by the quantum computer is at least as good as that of the classical computer for the problem we have studied.

This paper is organized as follows. In Section 2 we introduce the basic idea of quantum annealing. In Sections 3, 4, we present the formulation of a seismic inversion as a binary optimization problem and the methods used, respectively. In Section 5, the results obtained in the D-Wave quantum annealer are shown. In the last section, we draw the conclusion.

\section{QUANTUM ANNEALING}

In the literature, there are many different quantum computational models developed. Currently, three main models of quantum computing are being considered: The logic gate model, or circuit model, boson sampling, and the adiabatic model. The gate model is an universal quantum computation model that is performed programming a step-by-step instruction build from basic building blocks, known as quantum gates, similar to the classical circuit model [16]. This model is exploited by companies such as IBM, Intel, Rigetti, IonQ, AQT, and Google. Boson sampling computers consists in sampling from the output distribution of bosons in a linear interferometer [8, 17]. There is strong evidence that such an experiment is hard to be simulated in classical computers, but it is efficiently solved by special purpose photonic chips. The adiabatic computation model $[18,19]$ is a model in which the computational problem is mapped into a Hamiltonian, in such a way that the solution of the computational problem is encoded in the ground state of the quantum system represented by the Hamiltonian $H_{\text {final }}$. The computation is performed starting from the ground state of a known Hamiltonian $H_{\text {initial }}$. The
Hamiltonian is slowly modified towards to the target Hamiltonian $H_{\text {final }}$. During the process, the total Hamiltonian of the system is given by

$$
H(p)=(1-p) H_{\text {initial }}+p H_{\text {final }}
$$

where $p \in[0,1]$. According to the adiabatic theorem, if the evolution is performed adiabatically, the quantum state of the system remains in the instantaneous ground state throughout the entire process.

The adiabatic model is equivalent to the gate model [20], i.e., any problem that can be computed in the gate model will also be computable in the adiabatic model. This statement is valid for certain types of $k$ - local Hamiltonians [20]. For example, considering a system of $n$ qubits, we can perform universal adiabatic computation by choosing [21].

$$
\begin{aligned}
& H_{\text {initial }}=\sum_{i} \delta_{i} \sigma_{i}^{x}, \\
& H_{\text {final }}=\sum_{i} H_{i} \sigma_{i}^{z}+\sum_{j>i} J_{i, j} \sigma_{i}^{z} \sigma_{j}^{z}+\sum_{j>i} K_{i, j} \sigma_{i}^{x} \sigma_{j}^{x},
\end{aligned}
$$

where $\sigma_{i}^{k}$, with $k=x, y, z$, is one of the Pauli matrices of the $i^{\text {th }}$ qubit, $\delta_{i}$ and $H_{i}$ are local transverse and longitudinal fields, respectively, and $J_{i, j}$ and $K_{i, j}$ are coupling constants.

The adiabatic model can be viewed as a special case of the quantum annealing computing. In quantum annealers, the Hamiltonian change is not adiabatic. Therefore, quantum annealing is a heuristic type of computation. The D-Wave computer is a quantum annealer that uses Ising chains

$$
H_{\text {final }}=\sum_{i} H_{i} \sigma_{i}^{z}+\sum_{j>i} J_{i, j} \sigma_{i}^{z} \sigma_{j}^{z}
$$

The quantum annealing performed with Ising chains is unlikely to implement universal quantum computation [22]. Therefore, D-Wave annealers are more restrictive than the universal adiabatic model. Quantum annealing with Ising chains can be applied to a class of computational problems known as NP-hard problems [23]. It is believed that quantum annealing will be able to find better approximate solutions or find such approximate solutions faster then classical computers [22]. Here, it is also important to mention that the advantage of quantum annealers over classical methods is still under debate [24-26]. Recent works have proposed that, in some cases, quantum annealing is advantageous over classical computing [27-30], on the other hand, no advantage was reported in Refs. [24, 31]. The origin of the possible speedup is also under debate. Quantum tunneling is often claimed to be the key mechanism underlying possible speedups of quantum annealing. However, recent work has found numerical evidence that quantum tunneling processes can be efficiently simulated by Monte Carlo methods [32]. There is also evidence to suggest that it is unlikely to achieve exponential speedups over classical computing solely by the use of quantum tunneling [33]. The role of the temperature in the performance of quantum annealing has been also studied in [34].

To solve a problem in the D-Wave quantum annealer [6], it is necessary first to express the problem to be solved as an Ising 
problem or as a quadratic unconstrained binary optimization (QUBO), which is equivalent to Ising but defined on the binary values 0 and 1 , whereas the Ising problem is defined on the binary values $\pm 1 / 2$. The QUBO problem can be written as the minimization of the quadratic function

$$
f(\mathbf{q})=\mathbf{q}^{\top} \mathbf{Q q},
$$

where $\mathbf{q} \in\{0,1\}^{n}$, Q is a $n \times n$ upper (lower) triangular matrix and the vector $\mathbf{q}^{\top}=\left(q_{1}, q_{2}, \ldots, q_{n}\right)$ contains $n$ binary variables. QUBO problems are commonly used in machine learning and many important computational problems can be translated to a QUBO formulation as well [35]. Examples of problems that have been addressed with a $\mathrm{D}$-Wave quantum annealer are: the classification of human cancer types [36], traffic optimization [37], transcription factor DNA binding [29], metamaterial designing [38] and Higgs boson data analysis [39].

Recently, there has been a growing interest in quantum algorithms for systems of linear equations, $\mathbf{A x}=\mathbf{b}$, where $\mathbf{A}$ is a $n \times n$ matrix and $\mathbf{b}$ is a unit vector. Such algorithms may find applications in different research areas, including Geoscience. In the quantum gate model, the quantum version of such problem is called Quantum Linear Systems Problem (QLSP) [40, 41], and it is defined as the problem of preparing the state

$$
|\psi\rangle=\frac{\sum_{i}^{n} x_{i}|i\rangle}{\sqrt{\sum_{i}^{n}\left|x_{i}\right|^{2}}}
$$

where $\mathbf{x}=\left(x_{1}, x_{2}, \ldots\right)^{\top}$ is the solution of $\mathbf{A x}=\mathbf{b}$.

In 2008, Harrow, Hassidim, and Lloyd (HHL) proposed a quantum algorithm for the QLSP problem [42]. Given some assumptions [43], the run time of HHL is $O\left(k^{2} s^{2} \log (n) / \epsilon\right)$ where $k$ is the condition number of the matrix $\mathbf{A}$, defined as the absolute value of the ratio between the largest and smallest eigenvalues of $\mathbf{A}, s$ is the sparsity of $\mathbf{A}$, defined as the number of nonzero entries per row, and $\epsilon$ is the desired precision.

After the initial HHL proposal, several improvements were achieved: the condition number dependence was reduced from $k^{2}$ to $k \log ^{3}(k)$ [44], the error dependency was reduced from $1 /$ $\epsilon$ to a polynomial function in $\log (1 / \epsilon)$ [45], and a sparsityindependent runtime scaling was achieved in [46]. The QLSP problem can also be solved using iterative quantum solvers in runtime $O\left(k^{2} \log (k) / \log (1 / \epsilon)\right) \quad$ [47] and with runtimes $O(k \log (k) / \epsilon)$ and $O\left(k^{2} \log (k) / \epsilon\right)$ using the evolution randomization method, a simple variant of adiabatic quantum computing where the parameter $p$ in (1) varies discretely, rather than continuously [48]. The best generalpurpose classical conjugate gradient algorithm to solve $\mathbf{A x}=\mathbf{b}$ has the runtime $O(n k s \log (1 / \epsilon))$. Here, we must emphasize a fundamental difference between classical and quantum algorithms. While the conjugate gradient returns the solution vector $\mathbf{x}$, quantum algorithms return a quantum state, Eq. 6, that approximately contains all the components $x_{i}$ of the solution vector $\mathbf{x}$. It is possible to obtain any specific entry $x_{i}$ by measuring the output state (6), but in general, it will require repeating the algorithm many times, which would kill the exponential speedup. Still, quantum algorithms can be used as subroutines in different applications [40, 43].

In quantum annealers, the problems of solving a system of linear equations and a system of polynomial equations were previously studied in [49-51] and [52], respectively. Unlike the previously mentioned quantum algorithms, quantum annealing solves $\mathbf{A x}=\mathbf{b}$ completely, i.e., it returns the vector $\mathbf{x}$. To compare the performances of a quantum annealer and a classical computer, we must take into account the cost to prepare the problem, i.e., the procedure to map $\mathbf{A x}=\mathbf{b}$ into a QUBO or Ising problem, the cost to perform the annealing, and also the cost to post-process the results. The performance of quantum annealers to solve linear systems was studied in detail in Ref. [50]. It was shown that quantum annealers might be competitive if there exists a post-processing method that is polynomial in the size of the Matrix A with a degree less then 3.

\section{SEISMIC INVERSION WRITTEN AS A QUBO PROBLEM}

We considered the propagation of sound waves in a multi-layered medium, as shown in Figure 1. Multiple sources produce sound waves that can be reflected in the interface of each layer. Assuming that the wave propagation can be modeled as narrow beams or rays, the sound trace originated in the $i^{\text {th }}$ source reaches the $i^{\text {th }}$ detector after the time interval

$$
t_{i}=2 \sum_{j=1}^{i} \frac{d_{i j}}{v_{j}},
$$

where $d_{i j}$ and $v_{j}$ are the distance traveled by the sound waves and the sound speed in the $j^{\text {th }}$ layer, respectively. If we consider the thickness of each layer as $h_{j}$ and the distance between two consecutive sources (detectors) as $\Delta_{i}$, we can write $d_{i j}=h_{j} /$ $\cos \theta_{j}$ where $\theta_{j}=\arctan \left(\Delta_{j} / h_{j}\right)$.

The layered model described above is commonly used in seismic explorations, either offshore or onshore [53]. In seismic experiments the goal is to determine the velocities $\left\{v_{j}\right\}$ from the time intervals $\left\{t_{i}\right\}$, by solving the system

$$
\mathbf{M s}=\mathbf{t},
$$

where $\mathbf{t}^{\top}=\left(t_{1}, t_{2}, \ldots, t_{m}\right), \quad \mathbf{s}^{\top}=\left(1 / v_{1}, 1 / v_{2} \ldots, 1 / v_{m}\right)$ is the slowness vector, $\mathbf{M}$ is a $m \times m$ lower triangular matrix with nonzero elements given by $M_{., j}=2 h_{j} / \cos \theta_{j}$, and $m$ is the number of layers.

In order to use a quantum annealer to solve the above seismic problem it is necessary to translate the problem into a QUBO formulation. To proceed, first, we rewrite the system (8) as a minimization problem with the objective function

$$
f(\mathbf{s})=\|\mathbf{M s}-\mathbf{t}\|^{2},
$$

where $\mathbf{s} \in \mathbb{R}^{m}$. Next we write the slowness vector as $\mathbf{s}=\mathbf{s}_{\mathbf{0}}+L(\mathbf{x}-$ I), where $L$ defines the bounding limits of $\mathbf{s}, 0 \leq x_{i}<2 \forall x_{i}$, $\mathbf{I}^{\top}=(1,1, \ldots)$ and the vector $\mathbf{s}_{\mathbf{0}}$ is an initial guess for $\mathbf{s}$. The objective function $f(\mathbf{s})$ is rewritten as 


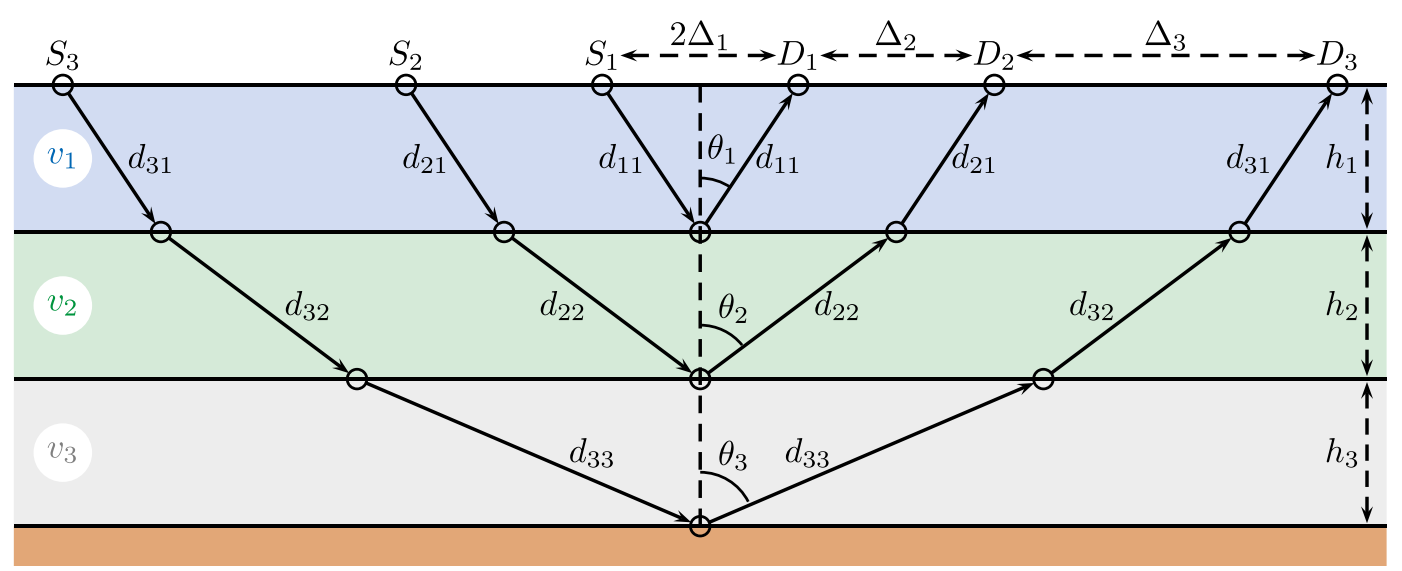

FIGURE 1 | Schematics of seismic data acquisition. Sound traces created in the $i^{\text {th }}$ source $S_{i}$ are detected at $D_{i}$ after the time of travel $t_{i}$. This figure depicts a simplified scheme with only $m=3$ layers, but we have considered up to $m=46$.

$$
f(\mathbf{x})=\|\mathbf{M x}-\mathbf{b}\|^{2},
$$

where $\mathbf{x} \in \mathbb{R}^{m}$ and $\mathbf{b}=\left(\mathbf{t}+L \mathbf{M I}-\mathbf{M} \mathbf{s}_{\mathbf{0}}\right) / L$. The matrix $\mathbf{M}$ and the vector $\mathbf{b}$ are parameters of the objective function while the vector $\mathbf{x}$ must be converted into a binary format. Here we discretized each $x_{i}$ variable with the R-bit approximation

$$
x_{i}=\sum_{r=0}^{R-1} q_{i, r} 2^{-r} .
$$

To formulate our QUBO problem, we construct a new binary vector $\mathbf{q}$ and a new real matrix $\mathbf{A}$ in order to form the binary system of equations $\mathbf{A q}=\mathbf{b}$. It is straightforward to reformulate this system as a binary optimization problem [49-51], whose solution vector, $\mathbf{q}^{\star}$, is given by

$$
\begin{aligned}
\mathbf{q} * & =\arg \min _{\mathbf{q} \in\{0,1\}^{R \times m}}\|\mathbf{A q}-\mathbf{b}\|^{2} \\
& =\arg \min _{\mathbf{q} \in\{0,1\}^{R \times m}} \mathbf{q}^{\top} \mathbf{Q} \mathbf{q}+C,
\end{aligned}
$$

where

$$
\begin{aligned}
Q_{i i} & =\sum_{k=1}^{m} A_{k i} A_{k i}-2 A_{k i} b_{k}, \\
Q_{i j} & =\sum_{k=1}^{m} 2 A_{k i} A_{k j}, \text { for } i<j,
\end{aligned}
$$

and $C=\sum_{k=1}^{m} b_{k}^{2}$ is an additive constant that does not change the ground state.

The precision of the solution depends on how many binary digits are used to represent the real variables of the problem, a solution with good precision would consume a large number of qubits of the quantum hardware. Here we have used a recursive approach similar to what was used in [49] to improve the precision of floating-point division. Our recursive approach is described in the Algorithm 1, using such an approach we could improve the solution of a system of linear equations with 46 real variables, using just a few qubits to represent each variable. Next, we will show in our example that using $R=3$, in Eq. 11, and carrying 20 iterations is sufficient to reach a good solution.

Algorithm 1. Function to solve the system of equations $\mathbf{M s}=\mathbf{t}$. The vector $\mathbf{s}_{\mathbf{0}}$ is the initial guess for $\mathbf{s}, \epsilon$ is the tolerance, $N_{\max }$ is the maximum number of iterations, and $L$ defines the interval where we expect to find the solutions (see text).

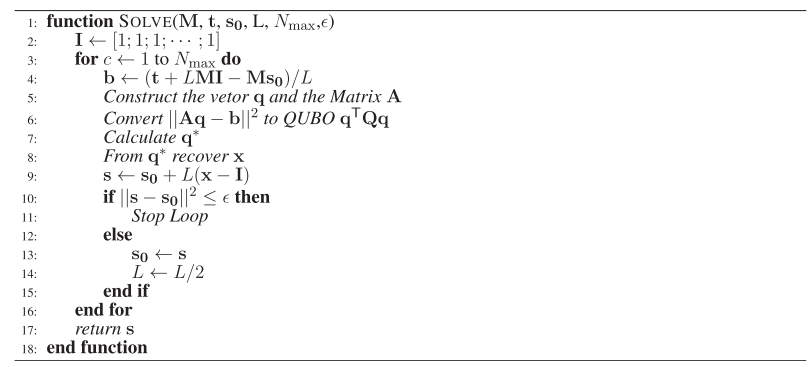

\section{METHODS}

We have performed the quantum computation with the D-Wave computer provided by the Amazon Braket service. Currently, there are two versions of quantum annealer available in Amazon Braket. The first is the D-wave 2000Q version, this computer contains 2041 working qubits. The connections among the qubits are represented by a graph called Chimera [54]. In this topology, each qubit is coupled to no more than 6 other qubits. We can call Chimera as a graph of degree 6. The second version is the D-Wave Advantage system, it is a more advanced computer with 5,436 working qubits disposed in a Pegasus graph with degree 15 [55]. A QUBO problem can also is represented by a graph, where each vertex of the graph corresponds to a binary variable $q_{i}$. When the QUBO problem is represented by a graph with degree greater then 6, for Chimera, or 15, for Pegasus, it is necessary to embed the QUBO graph onto the chip topology. The present seismic problem, for example, is represented by a full 

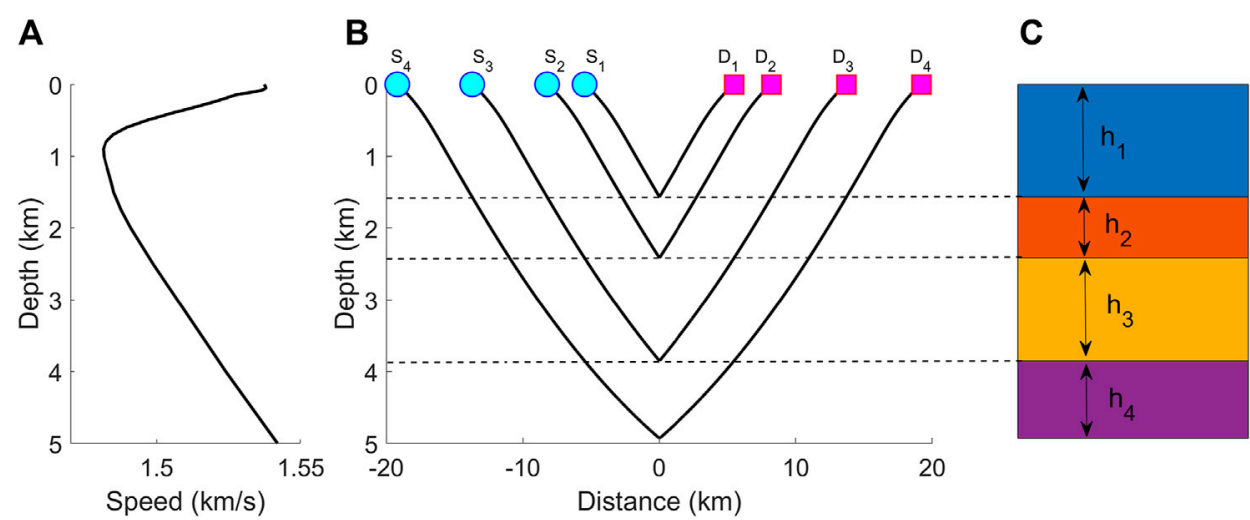

FIGURE 2 |Acoustic sound traces. In the (A), we show the sound speed profile of the Philippine sea [60]. In the (B), we show the results of numerical simulations of sound traces with incident angle $\theta_{0}=80^{\circ}$ and, in the $(\mathbf{C})$, we show the seismic model used. The thickness of each layer of the model matches the position where the corresponding sound trace is reflected. For clarity, we only show the simulations involving only 4 sources (receptors).
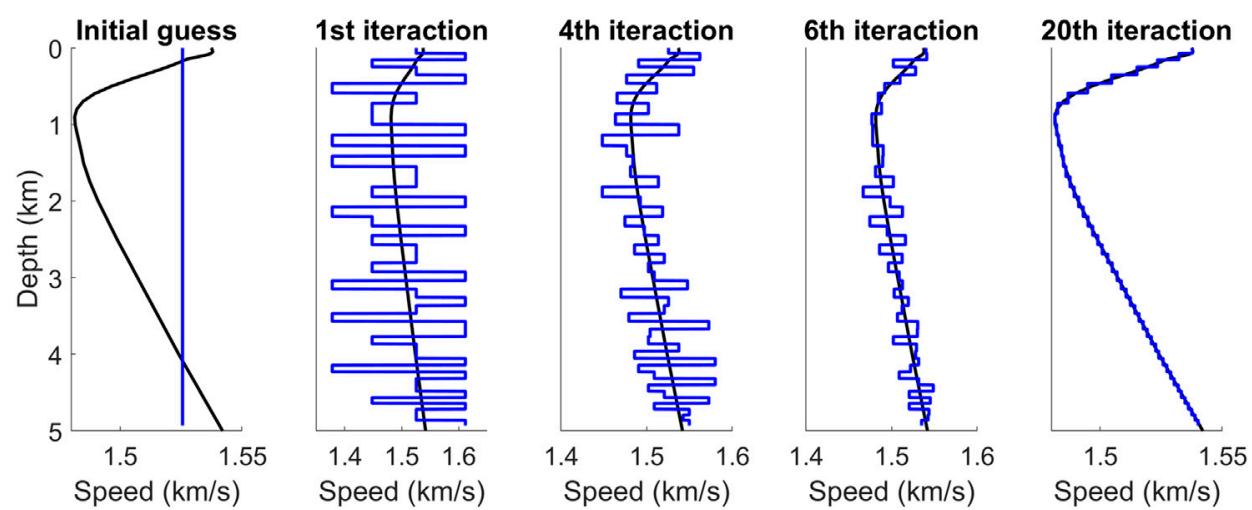

FIGURE 3 | Seismic inversion obtained in the quantum annealer for different iterations. The black curve is the original sound speed profile and the blue curve is the result of the inversion obtained in the quantum annealer.

graph, where each vertex is connected to all other vertices. In this work, the embeddings were obtained using a heuristic algorithm [56] provided by D-Wave.

Errors during the computation are important issues to be considered. In quantum annealing, the computational problem is encoded into the ground state of the Ising Hamiltonian (4), the gap between the ground state and the excited states is a key property of the system. If the gap is too small, thermal excitations and non-adiabatic transitions can induce transitions, as a consequence, the computer will output an excited state, which can be viewed as a computational error [57]. In addition, the wrong implementation of the Hamiltonian (4) may result in the wrong ground state [57]. We have noticed that the ground state was achieved with high probability. Therefore, unwanted transitions due to thermal fluctuations or nonadiabatic evolution do not represent an important issue in our case. The annealing time used was $20 \mu \mathrm{s}$, the minimal and default value of the $\mathrm{D}$-Wave machine. To post-process the output we use default D-Wave' routines.
In our implementation, analog errors are the most important, i.e., inaccurate implementations of the parameters $H_{i}$ and $J_{i, j}$ described in the Eq. 4. To reduce the impact of analog errors, we have used 10 spin-reversal transforms, also known as gauge transformations [58, 59]. This type of transformation is based on the fact that the structure of the Ising problem is not affected when the following transformations are applied: $H_{i} \rightarrow g_{i} H_{i}$ and $J_{i, j}$ $\rightarrow g_{i} g_{j} J_{i, j}$, where $g_{i}= \pm 1$. The original and transformed problems have identical energies. However, the sample statistics are affected by the spin-reversal transform because the quantum hardware is a physical object subject to errors.

\section{RESULTS}

We have applied the above formulation to solve a small scale underwater seismic inversion problem. Artificial data were generated by simulating sound traces in ocean. We have used the sound speed profile of the Philippine Sea, available to public [60], as shown in Figure 2. From the simulation, we obtained the 


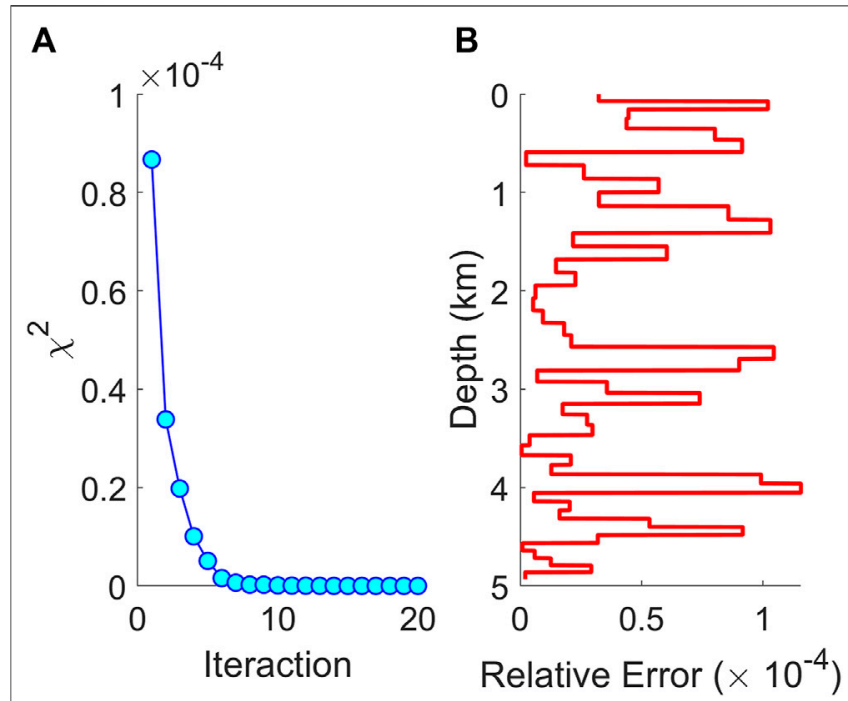

FIGURE 4 | Comparisons between classical and quantum solutions. In the (A), we show the $\chi^{2}$ test between the quantum annealing solution and the classical solution. The relative error between the classical and quantum solutions is shown as a function of the ocean depth in the (B)

travel times between the sources and detectors. The seismic inversion model was constructed using 46 layers while the real variables were digitalized with $R=3$ bits. This model yields 138 binary variables, for the present type of problem, it is the maximum number of variables that we can embed in the working graph of the D-Wave Advantage system available in Amazon.

To perform the seismic inversion we have considered that all layers in the model match the position where the sound wave is reflected, as shown in Figure 2. The solution is presented in Figure 3, as can be seen, good results are obtained with 20 iteractions. We have also performed the inversion with a classical computer running the forward substitution algorithm to invert the lower triangular matrix in Eq. 8. When classical and quantum inversions are compared, we found that the relative error between them was $\approx 10^{-4}$, as shown in Figure 4. This result shows that the quantum computer using the recursive approach to solve a system of linear equations has enough control to find solutions with good precision.

The results could also be compared to the benchmark provided by the condition number of the numerical problem at hand. When performing a numerical inversion procedure on the lower triangular matrix $\mathbf{M}$ to recover the solution $\mathbf{s}$ in Eq. 8, a straightforward estimation of the lower bound of the relative error $\epsilon_{s}$ in $\mathbf{s}$ arising from the relative error $\epsilon_{t}$ in the righthand side vector $\mathbf{t}$ due to the numerical conditioning of $\mathbf{M}$ is given by [61].

$$
\epsilon_{s}=\kappa_{\infty} \epsilon_{t},
$$

where $\kappa_{\infty}$ is the condition number given by

$$
\kappa_{\infty} \geq \frac{\max _{j}\left(h_{j} / \cos \theta_{j}\right)}{\min _{j}\left(h_{j} / \cos \theta_{j}\right)} .
$$

Therefore, the condition number relates the error associated with $\mathbf{t}, \epsilon_{s}$, and the error of the solution $\mathbf{s}, \epsilon_{s}$. In this particular application of seismic inversion, where distances are generally in the scale of $10^{3} \mathrm{~m}$, it is reasonable to assume that the figures are accurate up to the order of $\approx 10 \mathrm{~m}$, with the next order of magnitude $(1 \mathrm{~m})$ giving the scale of the error $\epsilon_{t}$. Given that the error $\epsilon_{t}$ can be estimated as $\approx 10^{-3}$ and the condition number is $\kappa_{\infty} \approx 1$, then we can estimate $\epsilon_{s} \approx 10^{-3}$ from (16). This shows that the error of the quantum approach comes from the inversion problem by itself and is of the same order as the classical approach.

\section{CONCLUSION}

Quantum computing represents a fundamentally different paradigm, an entirely different way to perform calculations. The Geoscience field and related industries are strong candidates to benefit from it. However, the performance of Geoscience inversion problems on current available quantum computers has so far been largely unexploited.

In this paper, we have solved a seismic inversions in a D-Wave quantum annealer. The seismic problem was written as a system of linear equations and then translated into a QUBO formulation. The results presented here indicate that the current available quantum annealers can solve a seismic inversion at a relatively small size with nearly the same accuracy as a classical computer. The proof-of-principle computations performed here show some promise for the use of quantum annealing in Geosciences.

The practical use of quantum annealing in Geosciences will require the ability to solve large problems. To address this issue, decomposer tools have been proposed to divide a large problem into small subproblems which can be solved individually by the quantum hardware [62-64]. Using such approach, it is possible to solve a large-sized problem using just a limited number of available qubits. Another interesting approach is the reverse annealing. Within this method, one starts from known local solutions which can be obtained in a classical computer. The annealing is performed backward from the known classical state to a state of quantum superposition, then proceeding forward it is possible to reach a new classical state that is a better solution than the initial one. Recently, it has been shown that it is possible to refine local solutions with recursive applications of reverse annealing [65-68]. We believe that the development of hybrid quantum-classical methods, such as mentioned above, will be essential to solve complex seismic problems on quantum annealers in the near future.

Finally, we should mention that the problem solved here is wellconditioned, however, often Geoscience problems are ill-conditioned. An interesting question is whether quantum computers can solve illconditioned problems efficiently. In Ref. [69], it was theoretically proposed that preconditioning methods can expand the number of linear systems problems that can achieve exponential speedup over classical computers. In future works, we plan to study the performance of the quantum annealers to solve ill-conditioned problems. Another attractive prospect for future work is the implementation of Geoscience problems in gate model computers, based either on superconducting or trapped ions technologies. 


\section{DATA AVAILABILITY STATEMENT}

Publicly available datasets were analyzed in this study. This data can be found here: Hodges RP. Underwater Acoustics (Wiley) (2002). (text book).

\section{AUTHOR CONTRIBUTIONS}

AS ran the quantum computer, analyzed the results, co-wrote, and reviewed the manuscript. EM initiated the study, co-wrote, and reviewed the manuscript. NS, IR, RS, and IO co-wrote and reviewed the manuscript.

\section{FUNDING}

This work was supported by the Brazilian National Institute of Science and Technology for Quantum Information (INCT-IQ)

\section{REFERENCES}

1. Arute F, Arya K, Babbush R, Bacon D, Bardin JC, Barends R, et al. Quantum Supremacy Using a Programmable Superconducting Processor. Nature (2019) 574:505-10. doi:10.1038/s41586-019-1666-5

2. Zhong H-S, Wang H, Deng Y-H, Chen M-C, Peng L-C, Luo Y-H, et al. Quantum Computational Advantage Using Photons. Science (2020) 370: 1460-3. doi:10.1126/science.abe8770

3. Wu Y, Bao W-S, Cao S, Chen F, Chen M-C, Chen X, et al. Strong Quantum Computational Advantage Using a Superconducting Quantum Processor. Phys Rev Lett (2021) 127:180501. doi:10.1103/ PhysRevLett.127.180501

4. Kjaergaard M, Schwartz ME, Braumüller J, Krantz P, Wang JI-J, Gustavsson S, et al. Superconducting Qubits: Current State of Play. Annu Rev Condens Matter Phys (2020) 11:369-95. doi:10.1146/annurev-conmatphys-031119-050605

5. Bruzewicz CD, Chiaverini J, McConnell R, Sage JM. Trapped-ion Quantum Computing: Progress and Challenges. Appl Phys Rev (2019) 6:021314. doi:10.1063/1.5088164

6. Job J, Lidar D. Test-driving 1000 Qubits. Quan Sci. Technol. (2018) 3:030501. doi:10.1088/2058-9565/aabd9b

7. Bourassa JE, Alexander RN, Vasmer M, Patil A, Tzitrin I, Matsuura T, et al. Blueprint for a Scalable Photonic Fault-Tolerant Quantum Computer. Quantum (2021) 5:392. doi:10.22331/q-2021-02-04-392

8. Brod DJ, Galvao EF, Crespi A, Osellame R, Spagnolo N, Sciarrino F. Photonic Implementation of Boson Sampling: a Review. Adv Photon (2019) 1:034001.

9. Linke NM, Maslov D, Roetteler M, Debnath S, Figgatt C, Landsman KA, et al. Experimental Comparison of Two Quantum Computing Architectures. Proc Natl Acad Sci USA (2017) 114:3305-10. doi:10.1073/pnas.1618020114

10. Devitt SJ. Performing Quantum Computing Experiments in the Cloud. Phys Rev A (2016) 94:032329. doi:10.1103/physreva.94.032329

11. O’Malley D. An Approach to Quantum-Computational Hydrologic Inverse Analysis. Sci Rep (2018) 8:6919. doi:10.1038/s41598-018-25206-0

12. Golden JK, O'Malley D. Pre- and post-processing in Quantum-Computational Hydrologic Inverse Analysis. Quan Inf Process (2021) 20:176. doi:10.1007/ s11128-021-03115-y

13. Alulaiw B, Sen MK. Prestack Seismic Inversion by Quantum Annealing: Application to Cana Field. In: SEG Technical Program Expanded Abstracts (2015). p. 3507-3511. doi:10.1190/segam2015-5831164.1

14. Wei C, Zhu P-M, Wang J-Y. Quantum Annealing Inversion and its Implementation. Chin J Geophys (2006) 49:499-506. doi:10.1002/cjg2.860
Grant No. 465469/2014-0, the Coordenação de Aperfeiçoamento de Pessoal de Nível Superior-Brasil (CAPES)-Finance Code 001, Conselho Nacional de Desenvolvimento Científico e Tecnológico (CNPq) and PETROBRAS: Projects 2017/00 486-1, 2018/00 233-9, and 2019/00 062-2. AS acknowledges support from FAPERJ (Grant No. 203.166/2 017). IO acknowledges FAPERJ (Grant No. 202.518/2 019).

\section{ACKNOWLEDGMENTS}

We are in debt with Prof. Daniel Lidar and the Information Sciences Institute of the University of Southern California, for giving us access to the D-Wave Quantum Annealer that was used in the first version of this work. The authors also acknowledges L. Cirto, N. L. Holanda and M.D. Correia for discussions and suggestions in the early stages of this work.

15. Greer S, O’Malley D. An Approach to Seismic Inversion with Quantum Annealing. In: SEG Technical Program Expanded Abstracts (2020). p. 2845-2849. doi:10.1190/segam2020-3424413.1

16. Nielsen MA, Chuang IL. Quantum Computaion and Quantum Information. Cambridge University Press (2001).

17. Aaronson S, Arkhipov A. The Computational Complexity of Linear Optics. In: Proceedings of the forty-third annual ACM symposium on Theory of computing (2011). p. 333. doi:10.1145/1993636.1993682

18. Farhi E, Goldstone J, Gutmann S, Lapan J, Lundgren A, Preda D. A Quantum Adiabatic Evolution Algorithm Applied to Random Instances of an Np-Complete Problem. Science (2001) 292:472-5. doi:10.1126/ science. 1057726

19. Albash T, Lidar DA. Adiabatic Quantum Computation. Rev Mod Phys (2018) 90:015002. doi:10.1103/revmodphys.90.015002

20. Aharonov D, van Dam W, Kempe J, Landau Z, Lloyd S, Regev O. Adiabatic Quantum Computation Is Equivalent to Standard Quantum Computation. SIAM J Comput (2007) 37:166-94. doi:10.1137/s0097539705447323

21. Biamonte JD, Love PJ. Realizable Hamiltonians for Universal Adiabatic Quantum Computers. Phys Rev A (2008) 78:012352. doi:10.1103/ physreva.78.012352

22. Preskill J. Quantum Computing in the Nisq Era and beyond. Quantum (2018) 2:79. doi:10.22331/q-2018-08-06-79

23. Barahona F. On the Computational Complexity of Ising Spin Glass Models. J Phys A: Math Gen (1982) 15:3241-53. doi:10.1088/0305-4470/15/10/028

24. Rønnow TF, Wang Z, Job J, Boixo S, Isakov SV, Wecker D, et al. Quantum Computing. Defining and Detecting Quantum Speedup. Science (2014) 345: 420-4. doi:10.1126/science.1252319

25. Mandra S, Zhu Z, Wang W, Perdomo-Ortiz A, Katzgraber H. Strengths and Weaknesses of Weak-strong Cluster Problems: A Detailed Overview of StateOf-The-Art Classical Heuristics versus Quantum Approaches. Phys Rev A (2016) 94:022337. doi:10.1103/physreva.94.022337

26. Katzgraber H, Hamze F, Zhu Z, Ochoa A, Munoz-Bauza H. Seeking Quantum Speedup through Spin Glasses: The Good, the Bad, and the Ugly. Phys Rev X (2015) 5:031026. doi:10.1103/physrevx.5.031026

27. Albash T, Lidar DA. Demonstration of a Scaling Advantage for a Quantum Annealer over Simulated Annealing. Phys Rev X (2018) 8:031016. doi:10.1103/ physrevx.8.031016

28. Denchev V, Boixo S, Isakov S, Ding N, Babbush R, Smelyanskiy V, et al. What Is the Computational Value of Finite-Range Tunneling. Phys Rev X (2016) 6: 031015. doi:10.1103/physrevx.6.031015

29. Li RY, Di Felice R, Rohs R, Lidar DA. Quantum Annealing versus Classical Machine Learning Applied to a Simplified Computational Biology Problem. Npj Quan Inf (2018) 4:14. doi:10.1038/s41534-018-0060-8 
30. Baldassi C, Zecchina R. Efficiency of Quantum vs. Classical Annealing in Nonconvex Learning Problems. Proc Natl Acad Sci USA (2018) 115:1457-62. doi:10.1073/pnas.1711456115

31. Hen I, Job J, Albash T, Ronnow T, Troyer M, Lidar D. Probing for Quantum Speedup in Spin-Glass Problems with Planted Solutions. Phys Rev A (2015) 95: 042325. doi:10.1103/physreva.92.042325

32. Brady LT, van Dam W. Quantum Monte Carlo Simulations of Tunneling in Quantum Adiabatic Optimization. Phys Rev A (2016) 93:032304. doi:10.1103/ physreva.93.032304

33. Crosson E, Harrow AW. Simulated Quantum Annealing Can Be Exponentially Faster Than Classical Simulated Annealing. In: 2016 IEEE 57th Annual Symposium on Foundations of Computer Science (FOCS); 9-11 Oct. 2016; New Brunswick, NJ, USA. (2016) 714. doi:10.1109/focs.2016.81

34. Jiang Z, Smelyanskiy VN, Isakov SV, Boixo S, Mazzola G, Troyer M, et al. Scaling Analysis and Instantons for Thermally-Assisted Tunneling and Quantum Monte Carlo Simulations. Phys Rev A (2017) 95:012322. doi:10.1103/physreva.95.012322

35. Lucas A. Ising Formulations of many Np Problems. Front Phys (2014) 2:5. doi:10.3389/fphy.2014.00005

36. Li RY, Gujja S, Bajaj SR, Gamel OE, Cilfone N, Gulcher JR, et al. Quantum Processor-Inspired Machine Learning in the Biomedical Sciences. Patterns (2021) 2:100246. doi:10.1016/j.patter.2021.100246

37. Neukart F, Compostella G, Seidel C, von Dollen D, Yarkoni S, Parney B. Traffic Flow Optimization Using a Quantum Annealer. Front ICT (2017) 4:29. doi:10.3389/fict.2017.00029

38. Kitai K, Guo J, Ju S, Tanaka S, Tsuda K, Shiomi J, et al. Designing Metamaterials with Quantum Annealing and Factorization Machines. Phys Rev Res (2020) 2:013319. doi:10.1103/PhysRevResearch.2.013319

39. Mott A, Job J, Vlimant J-R, Lidar D, Spiropulu M. Solving a Higgs Optimization Problem with Quantum Annealing for Machine Learning. Nature (2017) 550:375-9. doi:10.1038/nature24047

40. Duan B, Yuan J, Yu C-H, Huang J, Hsieh C-Y. A Survey on HHL Algorithm: From Theory to Application in Quantum Machine Learning. Phys Lett A (2020) 384:126595. doi:10.1016/j.physleta.2020.126595

41. Dervovic D, Herbster M, Mountney P, Severini S, Usher N, Wossnig L. Quantum Linear Systems Algorithms: A Primer. $\operatorname{arXiv}$ [Preprint] (2018). Available from https://arxiv.org/abs/1802.08227.

42. Harrow AW, Hassidim A, Lloyd S. Quantum Algorithm for Linear Systems of Equations. Phys Rev Lett (2009) 103:150502. doi:10.1103/PhysRevLett.103.150502

43. Aaronson S. Read the fine Print. Nat Phys (2015) 11:291-3. doi:10.1038/nphys3272

44. Ambainis A. Variable Time Amplitude Amplification and Quantum Algorithms for Linear Algebra Problems. In: C Dürr T Wilke, editors. 29th International Symposium on Theoretical Aspects of Computer Science (STACS 2012), 14. Dagstuhl, Germany: Schloss Dagstuhl-Leibniz-Zentrum fuer Informatik (2012). p. 636-47. Leibniz International Proceedings in Informatics (LIPIcs). doi:10.4230/LIPIcs.STACS.2012.636

45. Childs AM, Kothari R, Somma RD. Quantum Algorithm for Systems of Linear Equations with Exponentially Improved Dependence on Precision. SIAM J Comput (2017) 46:1920-50. doi:10.1137/16M1087072

46. Wossnig L, Zhao Z, Prakash A. Quantum Linear System Algorithm for Dense Matrices. Phys Rev Lett (2018) 120:050502. doi:10.1103/PhysRevLett.120.050502

47. Shao C, Xiang H. Row and Column Iteration Methods to Solve Linear Systems on a Quantum Computer. Phys Rev A (2020) 101:022322. doi:10.1103/physreva.101.022322

48. Subasi Y, Somma RD, Orsucci D. Quantum Algorithms for Systems of Linear Equations Inspired by Adiabatic Quantum Computing. Phys Rev Lett (2019) 122:060404. doi:10.1103/PhysRevLett.122.060504

49. Rogers ML, Singleton RL. Floating-point Calculations on a Quantum Annealer: Division and Matrix Inversion. Front Phys (2020) 8:8. doi:10.3389/fphy.2020.00265

50. Borle A, Lomonaco SJ. Analyzing the Quantum Annealing Approach for Solving Linear Least Squares Problems. In: GK Das, PS Mandal, K Mukhopadhyaya, S Nakano, editors. WALCOM: Algorithms and Computation. Springer (2019). p. 289-301. doi:10.1007/978-3-030-10564-8_23

51. O'Malley D, Vesselinov VV. ToQ.jl: A High-Level Programming Language for D-Wave Machines Based on Julia. In: IEEE Conference on High Performance Extreme Computing. HPEC (2016). p. 1-7. doi:10.1109/HPEC.2016.7761616

52. Chang CC, Gambhir A, Humble TS, Sota S. Quantum Annealing for Systems of Polynomial Equations. Sci Rep (2019) 9:10258. doi:10.1038/s41598-019-46729-0
53. Kearey P, Brooks M, Hill I. An Introduction to Geophysical Exploration. 3 edn Wiley (2002).

54. Bunyk PI, Hoskinson EM, Johnson MW, Tolkacheva E, Altomare F, Berkley AJ, et al. Architectural Considerations in the Design of a Superconducting Quantum Annealing Processor. IEEE Trans Appl Supercond (2014) 24:1-10. doi:10.1109/TASC.2014.2318294

55. Dattani N, Szalay S, Chancellor N. Pegasus: The Second Connectivity Graph for Large-Scale Quantum Annealing Hardware. arXiv[Preprint] (2019). Available from https://arxiv.org/abs/1901.07636.

56. Cai J, Macready B, Roy A. A Practical Heuristic for Finding Graph Minors. $\operatorname{arXiv}[$ Preprint] (2014). Available from https://arxiv.org/abs/1406.2741.

57. Pearson A, Mishra A, Hen I, Lidar DA. Analog Errors in Quantum Annealing: Doom and hope. Npj Quan Inf (2019) Vol. 5:2347. doi:10.1038/s41534-0190210-7

58. [Dataset] Inc DWS. Qpu Solver Datasheet (2021). Available at: file:///C:/Users/alexa/ Downloads/09-1109B-C_QPU_Solver_Datasheet.pdf (Accessed October 15, 2021).

59. Pelofske E, Hahn G, Djidjev H. Optimizing the Spin Reversal Transform on the D-Wave 2000q2019 IEEE International Conference on Rebooting Computing (ICRC) (2019). p. 1-8. doi:10.1109/ICRC.2019.8914719

60. Hodges RP. Underwater Acoustics. Wiley (2002).

61. Kincaid D, Cheney W. Numerical Analysis: Mathematics of Scientific Computing. USA: Brooks/Cole Publishing (1991).

62. [Dataset] Inc DWS. Qbsolv Documentation (2021). Available at: https://docs. ocean.dwavesys.com/_/downloads/qbsolv/en/latest/pdf/ (Accessed October $15,2021)$.

63. Okada S, Ohzeki M, Terabe M, Taguchi S. Improving Solutions by Embedding Larger Subproblems in a D-Wave Quantum Annealer. Sci Rep (2019) 9:2098. doi:10.1038/s41598-018-38388-4

64. Nishimura N, Tanahashi K, Suganuma K, Miyama MJ, Ohzeki M. Item Listing Optimization for E-Commerce Websites Based on Diversity. Front Comput Sci (2019) 1:2. doi:10.3389/fcomp.2019.00002

65. Passarelli G, Yip KW, Lidar DA, Nishimori H, Lucignano P. Reverse Quantum Annealing of the P-Spin Model with Relaxation. Phys Rev A (2020) 101: 022331. doi:10.1103/physreva.101.022331

66. Yamashiro Y, Ohkuwaa M, Nishimori H, Lidar DA. Dynamics of Reverse Annealing for the Fully Connected P-Spin Model. Phys Rev A (2019) 100: 052331. doi:10.1103/physreva.100.052321

67. Venturelli D, Kondratyev A. Reverse Quantum Annealing Approach to Portfolio Optimization Problems. Quan Mach. Intell. (2019) 1:17-30. doi:10.1007/s42484-019-00001-w

68. Arai S, Ohzeki M, Tanaka K. Mean Field Analysis of Reverse Annealing for Code-Division Multiple-Access Multiuser Detection. Phys Rev Res (2021) 3: 033006. doi:10.1103/physrevresearch.3.033006

69. Clader BD, Jacobs BC, Sprouse CR. Preconditioned Quantum Linear System Algorithm. Phys Rev Lett (2013) 110:250504. doi:10.1103/ physrevlett.110.250504

Conflict of Interest: Author EM was employed by the company Petróleo Brasileiro S.A.

The remaining authors declare that the research was conducted in the absence of any commercial or financial relationships that could be construed as a potential conflict of interest.

Publisher's Note: All claims expressed in this article are solely those of the authors and do not necessarily represent those of their affiliated organizations, or those of the publisher, the editors and the reviewers. Any product that may be evaluated in this article, or claim that may be made by its manufacturer, is not guaranteed or endorsed by the publisher.

Copyright $\odot 2022$ Souza, Martins, Roditi, Sá, Sarthour and Oliveira. This is an openaccess article distributed under the terms of the Creative Commons Attribution License (CC BY). The use, distribution or reproduction in other forums is permitted, provided the original author(s) and the copyright owner(s) are credited and that the original publication in this journal is cited, in accordance with accepted academic practice. No use, distribution or reproduction is permitted which does not comply with these terms. 